\title{
Hallazgos radiográficos de connotación endodóntica utilizando tomografía computarizada de haz cónico
}

\section{Radiographic findings of endodontic connotation using cone beam computed tomography}

\author{
Monardes Cortés H*, Abarca Reveco J*, Chaparro González D, Pizarro Gamboa F*
}

\section{RESUMEN}

En la práctica de la odontología, la tomografía computarizada de haz cónico (TCHC) ha sido utilizada en la indicación de implantes y cirugía maxilofacial. En endodoncia, ya ha comenzado su uso, por la posibilidad de conocer las tres dimensiones del diente.

Este estudio busca determinar la frecuencia de las diferentes condiciones patológicas más prevalentes en endodoncia, mediante el uso de TCHC, para lo cual se identificaron y cuantificaron hallazgos radiográficos de connotación endodóntica en 128 TCHC en pacientes chilenos entre 7 y 78 años, de ambos sexos, evaluando un total de 1.213 dientes. Las imágenes fueron obtenidas por un equipo SkyView 3D TCHC Panoramic Imager ${ }^{\circledR} 12$ bits (MyRay, Imola, Italia), con una exposición de $90 \mathrm{Kvp}, 10 \mathrm{~mA}$ y observadas mediante el software Skyview viewer manager ver 1.2.0.6 MyRay por 2 observadores previamente calibrados. Se observaron los dientes de manera independiente, en los ejes sagital, coronal y axial con un intervalo de corte de 0,5 $\mathrm{mm}$. Los datos se introdujeron en la versión 17.0 del paquete estadístico SPSS ${ }^{\circledR}$ para Windows (SPSS Inc., Chicago, IL) para su análisis.

Los hallazgos de connotación endodóntica fueron: lesión apical, terapia endodóntica realizada, taurodontismo, canales accesorios, dilaceraciones, ápices abiertos, reabsorción interna, reabsorción externa apical y cervical. Se encontró asociación significativa entre la presencia de lesiones apicales y dientes endodónticamente tratados $(p=0,00)$.

Palabras clave: Endodoncia, Tomografía Computarizada, hallazgos radiográficos.

\section{SUMMARY}

In the practice of dentistry, cone beam computed tomography (CBCT) has been used in the indication of implants and maxillofacial surgery. In endodontics its use has already begun, because of the possibility to learn the three dimensions of the tooth.

The aim of this study was to determine the frequency of various conditions that are the most prevalent in endodontics, using CBCT, for which they were identified and quantified radiographic findings of endodontic connotation in 128 CBCT in Chilean patients between 7 and 78 years old, of both sexes, evaluating a total of 1213 teeth. The images were obtained by SkyView 3D Panoramic CBCT Imager ${ }^{\circledR} 12$ bits (MyRay, Imola, Italy), with an exposure of $90 \mathrm{Kvp}, 10 \mathrm{~mA}$ and observed by software Skyview viewer manager ver 1.2.0.6 MyRay by two previously calibrated observers. Teeth were observed in the sagittal, coronal and axial axes with a cutting interval of $0.5 \mathrm{~mm}$. The data was introduced into" SPSS Statistics package for Windows, version 17.0) (SPSS Inc., Chicago, IL) for analysis.

* Curso de Especialización en Endodoncia. Facultad de Odontología. Universidad San Sebastián. Santiago, Chile. 
The findings of endodontic connotation were: apical lesion, endodontic therapy performed, taurodontism, accessory canals, dilacerations, open apices, internal resorption, cervical and apical external resorption). Significant association between the presence of endodontically treated teeth and apical lesions $(p=0.00)$ was found.

Key words: Endodontic, computed tomography, radiographic findings.

Fecha de recepción: 21 de octubre de 2014.

Aceptado para publicación: 15 de enero de 2015.

Monardes Cortés H, Abarca Reveco J, Chaparro González D, Pizarro Gamboa F. Hallazgos radiográficos de connotación endodóntica utilizando tomografía computarizada de haz cónicoo. Av. Odontoestomatol 2015; 31 (2): 59-65.

\section{INTRODUCCIÓN}

En la práctica de la odontología, la tomografía computarizada de haz cónico (TCHC) ha sido utilizada ampliamente, especialmente en la indicación de implantes y preparación de cirugía maxilofacial (1). La endodoncia no escapa a este fenómeno debido a que los especialistas que ya se han interiorizado en esta ayuda clínica han aumentado su uso.

Esto se debe a que la información que es posible recopilar en una película periapical convencional está limitada a una imagen bidimensional, donde además es probable que exista una distorsión geométrica de las estructuras anatómicas a examinar (2).

Gracias a la posibilidad de conocer en forma adecuada las tres dimensiones del diente, sumado a la ventaja de la TCHC en precisión hace que este examen se esté utilizando en casos de mayor complejidad en endodoncia (3).

El objetivo de este trabajo fue determinar la frecuencia de las diferentes condiciones patológicas más prevalentes en endodoncia, mediante el uso de TCHC.

\section{MATERIAL Y MÉTODOS}

Para la realización de este estudio descriptivo se utilizaron 128 tomografías computarizadas de haz cónico obtenidas de un archivo de exámenes solicitados con distintos fines durante el año 2011, en pacientes chilenos entre 7 y 78 años, de ambos sexos, cuyos nombres de los pacientes fueron codificados en el programa Excel ${ }^{\circledR}$ versión 12.0 para Windows (Microsoft Corporation) para mantener en secreto su identidad, tal como establece el acuerdo de Helsinki (4).

Las imágenes fueron obtenidas por un equipo SkyView 3D TCHC Panoramic Imager ${ }^{\circledR} 12$ bits (MyRay, Imola, Italia), con una exposición de 90 Kvp, $10 \mathrm{~mA}$, utilizando campos de visión pequeños, para el maxilar (FOV no mayor a 4 pulgadas) y/o mandíbula (FOV no mayor a 6 pulgadas). Se visualizaron las piezas dentarias de manera independiente, en los 3 ejes (sagital, coronal y axial) de cada una, con un intervalo de corte de $0,5 \mathrm{~mm}$ con un análisis multiplanar en de cada raíz. Las que fueron analizadas usando el software Skyview viewer manager ver 1.2.0.6 MyRay utilizando computadores portátiles (MacBook Pro, Apple Computer Inc.) con una pantalla 15,5 pulgadas (LED) retroiluminada con una resolución de $1.440 \times 900$, por 2 observadores previamente calibrados, que obtuvieron un grado de concordancia inter observador de 0,812.

Se excluyeron los dientes con pernos metálicos, errores en la imagen, en angulaciones y con restauraciones metálicas que afectaban la visualización correcta del diente, evaluando un total de 1.213 dientes. Los hallazgos de connotación endodóntica a observar fueron: lesión apical, terapia endodóntica, taurodontismo, canales accesorios, dilaceraciones, ápices abiertos y reabsorciones (6). 
Los datos obtenidos se introdujeron en la versión 17.0 del paquete estadístico SPSS ${ }^{\circ}$ para Windows (SPSS Inc., Chicago, IL) para su análisis.

\section{RESULTADOS}

Los distintos hallazgos de connotación endodóntica observados, en frecuencia y porcentaje, se encuentran tabulados en la tabla 1 y se ven en la figura 1 . La presencia de lesiones apicales es estadísticamente significativa con un $p=0,001$, con un intervalo de confianza de un $95 \%(\mathrm{p}<0,05)$ en relación los hallazgos radiográficos.

La presencia de lesiones apicales en el total de casos observados se ven en la tabla 2.

Los dientes que presentan terapia endodóntica del total de casos observados se ven en la tabla 3 .

Del total de dientes evaluados, los casos que presentaron reabsorción radicular, en cuanto a número y porcentaje, se ven en la tabla 4.

Con respecto a la presencia de lesiones apicales en dientes tratados endodónticamente, éstas se obser-

\section{TABLA 1.- HALLAZGOS RADIOGRÁFICOS DE CONNOTACIÓN ENDODÓNTICA}

\begin{tabular}{|l|c|c|}
\hline $\begin{array}{l}\text { Hallazgos } \\
\text { radiográficos }\end{array}$ & $\begin{array}{c}\text { Número } \\
\text { de casos }\end{array}$ & Porcentaje \\
\hline Lesión apical & 180 & 14,80 \\
Terapia endodóntica & 122 & 10,10 \\
Taurodontismo & 3 & 0,20 \\
Canales accesorios & 45 & 3,70 \\
Dilaceraciones & 111 & 9,20 \\
Ápices abiertos & 33 & 2,70 \\
Reabsorción interna & 3 & 0,20 \\
Reabsorción externa A & 36 & 3,00 \\
Reabsorción externa C & 3 & 0,20 \\
\hline
\end{tabular}

\section{TABLA 2.- PRESENCIA DE LESIÓN APICAL} EN EL TOTAL DE CASOS

\begin{tabular}{|r|c|c|}
\hline & $\begin{array}{c}\text { Número } \\
\text { de casos }\end{array}$ & $\begin{array}{c}\text { Porcentaje } \\
\text { de total }\end{array}$ \\
\hline Ausencia de lesión apical & 1.033 & 85,20 \\
Presencia de lesión apical & 180 & 14,80 \\
\hline Total & 1.213 & 100,00 \\
\hline
\end{tabular}

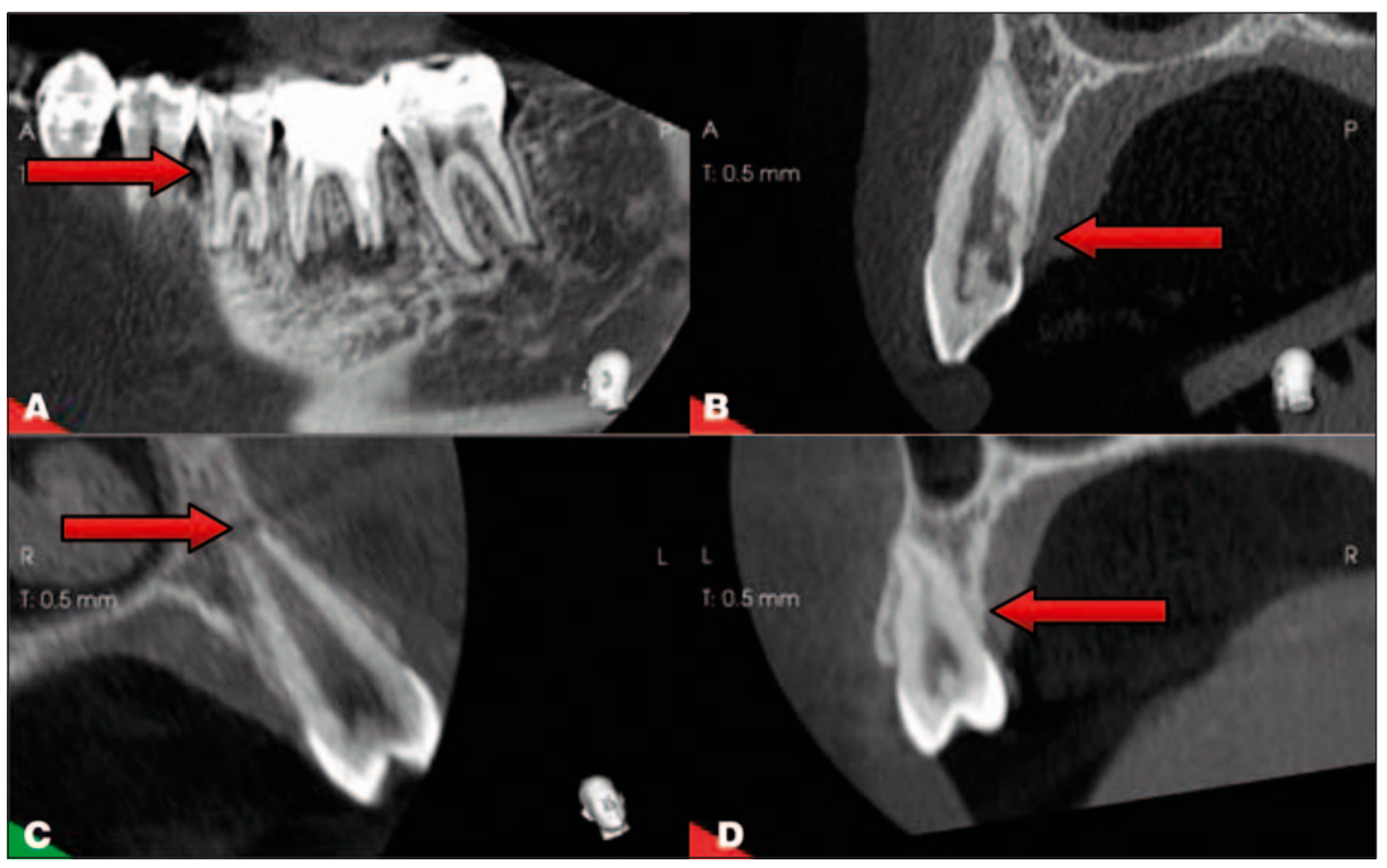

Fig.1. Hallazgos de connotación endodóntica. A) Taurodontismo.

B) Reabsorción interna.

C) Ápice abierto.

D) Dislaceracion radicular. 


\begin{tabular}{|c|c|c|}
\hline \multicolumn{3}{|c|}{$\begin{array}{l}\text { TABLA 3.- PRESENCIA DE TERAPIA } \\
\text { ENDODÓNTICA EN EL TOTAL DE CASOS }\end{array}$} \\
\hline & $\begin{array}{l}\text { Número } \\
\text { de casos }\end{array}$ & $\begin{array}{c}\text { Porcentaje } \\
\text { de total }\end{array}$ \\
\hline $\begin{array}{l}\text { Presencia de terapia } \\
\text { endodóntica } \\
\text { Ausencia de terapia } \\
\text { endodóntica }\end{array}$ & 1.091 & 89,90 \\
\hline Total & 1.213 & 100,00 \\
\hline
\end{tabular}

\section{TABLA 4.- REABSORCIONES RADICULARES} EN EL TOTAL DE CASOS

\begin{tabular}{|l|r|r|}
\hline & $\begin{array}{c}\text { Número } \\
\text { de casos }\end{array}$ & $\begin{array}{r}\text { Porcentaje } \\
\text { de total }\end{array}$ \\
\hline $\begin{array}{l}\text { No se observa } \\
\text { reabsorción } \\
\begin{array}{l}\text { Reabsorción interna } \\
\text { Reabsorción externa } \\
\text { apical }\end{array}\end{array}$ & 1.171 & 96,50 \\
$\begin{array}{l}\text { Reabsorción externa } \\
\text { cervical }\end{array}$ & 3 & 0,20 \\
\hline Total & 1.213 & 3,00 \\
\hline
\end{tabular}

van en la tabla 5 y se visualizan en la figura 2 . La prueba de chi-cuadrado, a un intervalo de confianza del 95\% ( $<<0,05)$, demostró un valor de $p=0,000$; por lo tanto, la presencia de lesiones apicales es estadísticamente significativa en piezas endodónticamente tratadas.

\section{DISCUSIÓN}

Entre las principales indicaciones de la TCHC en la práctica endodóntica, se pueden enumerar: incor-

\begin{tabular}{|r|c|c|}
\hline \multicolumn{3}{|c|}{ TABLA 5.- PRESENCIA DE LESIÓN APICAL EN } \\
DIENTES TRATADOS ENDODÓNTICAMENTE \\
\hline & $\begin{array}{l}\text { Piezas tratadas } \\
\text { endodónticamente }\end{array}$ & Porcentaje \\
\hline $\begin{array}{l}\text { Presencia de lesión } \\
\text { apical } \\
\begin{array}{l}\text { Ausencia de lesión } \\
\text { apical }\end{array}\end{array}$ & 86 & 70,49 \\
\hline Total & 122 & 100,00 \\
\hline
\end{tabular}

Fig. 2. Lesiones apicales asociadas a tratamientos endodónticos.
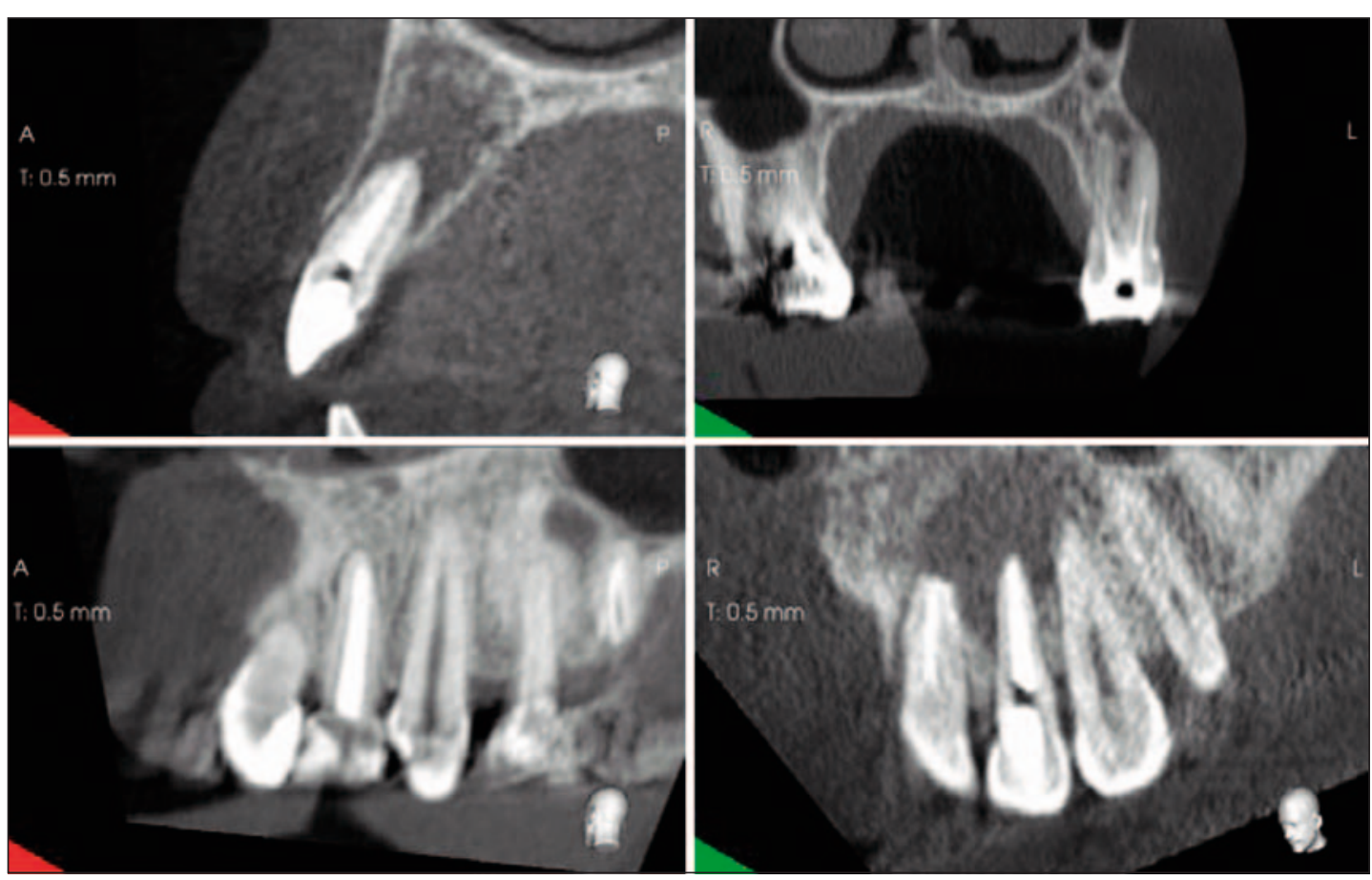
porar nueva información que no estaba disponible con otros métodos; reducir la radiación absorbida por el paciente; obtener la mayor resolución posible y disponer de múltiples campos de observación (7).

Se han descrito diversas aplicaciones en endodoncia de la TCHC: identificación de canales accesorios; identificación de las anomalías del sistema de canales radiculares y la determinación de curvatura radicular; diagnóstico de patología periapical en los pacientes que presentan signos clínicos contradictorios o síntomas no específicos; en los casos de superposición anatómica de las raíces o áreas del esqueleto; diagnóstico de patología de origen no endodóntico con el fin de determinar la extensión de la lesión; evaluación de las complicaciones del tratamiento de endodoncia; identificación del canal calcificado, y localización de perforaciones; diagnóstico de traumatismos dentoalveolares; determinación de reabsorciones radiculares internas, externas, cervicales; y determinación de la ubicación exacta del ápice del ápice radicular para evaluar la proximidad de las estructuras anatómicas adyacentes (8-11).

La presencia de dilaceraciones en endodoncia presenta un desafío para el operador clínico tanto en el diagnóstico, como en el tratamiento (12), dependiendo de la severidad de la curvatura (13). En nuestro estudio, las dilaceraciones alcanzaron al 9,2\% del total de dientes examinados, porcentaje coincidente con estudios de población adulta en Croacia (14). Otras anomalías fueron encontradas en muy bajo porcentaje, raíces y canales accesorios $(3,7 \%)$.

Taurodontismo y canales en $\mathrm{C}$ fueron encontrados en un bajísimo porcentaje (un 0,2\% del total de los dientes observados) que corresponde al 0,91\% de los molares observados, concordando con reportes en la literatura (15-17).

Esta es una probabilidad mínima de aparición, pero el canal en forma de $C$ es un desafío para el desbridamiento y la obturación (17). Los dientes con taurodontismo muestran variaciones en el tamaño y forma de las cámaras pulpares, diversos grados de complejidad del canal, piso cameral y mayor predisposición a la existencia de canales accesorios (16).
El ápice abierto representa solo un 2,7\% de los dientes estudiados y siempre es un factor a considerar ya que el manejo de estos dientes es la apexificación o bien la apexogénesis (18).

El uso de TCHC es altamente preciso para la detección de las reabsorciones tanto interna como externa, tal como ha sido ampliamente demostrado en distintos estudios (19-21). Estas se presentaron en solo un $3,5 \%$, siendo la más común la reabsorción externa apical.

Es trascendente la prevalencia de patología periapical en dientes con tratamiento endodóntico, según los estudios Toronto (22), el porcentaje de éxito endodóntico en dientes vitales es del $93 \%$, y en dientes con periodontitis apical es de $78 \%$, ambas con tratamiento óptimo, esto determinado con radiografía periapical e índice periapical (23).

La TCHC ha demostrado ser una herramienta muy poderosa en la detección de lesiones apicales debido a que la imagen se representa tridimensionalmente en los ejes del espacio, y excluye estructuras anatómicas que interfieren en la visión normal de la lesión. El clínico puede observar cortes específicos de la raíz y la locación de la lesión, algo prácticamente imposible de detectar con otros medios como la radiografía retroalveolar (24-26).

En el presente estudio, se observó lesión apical en un $70,49 \%$ del total de dientes tratados endodónticamente, lo que coincide con otro reporte (27), en el cual encontraron presencia de signos patológicos periapicales en el 60,9\% de los casos estudiados. En ambos estudios, la cantidad de muestras fue similar.

Existen diferencias en determinar el éxito de la terapia endodóntica mediante el uso de TCHC y radiografía periapical, pues diferentes estudios han demostrado la mayor sensibilidad de la TCHC para detectar signos de patología apical, pues su sensibilidad es de un $100 \%$ versus un $25 \%$ de las radiografías periapicales (28). Asimismo, otro estudio analizó histológicamente en perros, lesiones periapicales artificialmente creadas que solo eran visibles en TCHC y no en radiografía periapical, demostrando tener más sensibilidad que la radiografía periapical 
(9). Además, TCHC es efectiva para evaluar la densidad ósea en zonas maxilares relacionadas con tratamientos endodónticos (29).

En el presente trabajo, se logró identificar numerosos descubrimientos de relativos a la endodoncia, estos hallazgos concuerdan con los encontrados en estudios similares anteriores. Se logró establecer una relación entre la presencia de lesiones apicales y tratamientos endodónticos. Esta relación es mayor a la encontrada en estudios realizados con radiografías convencionales, pero concuerdan con estudios realizados con TCHC. En el caso de hallazgos de tipo anatómicos los datos recogidos concuerdan con la literatura.

Es necesario profundizar la investigación del diagnóstico endodóntico mediante TCHC, pues no ha sido llevada a todo su potencial en el área de la endodoncia y la posible información a recopilar puede ser de suma importancia para el diagnóstico, tratamiento y determinación del pronóstico de la terapia endodóntica.

\section{BIBLIOGRAFÍA}

1. Scarfe WC, Farman AG, Sukovic P. Clinical applications of cone-beam computed tomography in dental practice. Journal of the Canadian Dental Association 2006;72(1):75-80.

2. Lofthag-Hansen S, Huumonen S, Grondahl K, Grondahl HG. Limited cone-beam CT and intraoral radiography for the diagnosis of periapical pathology. Oral Surgery, Oral Medicine, Oral Pathology, Oral Radiology and Endodontology 2007;103(1):114-9.

3. Scarfe W C, Farman A G, Levin M D, Gane D. Essentials of maxillofacial cone beam computed tomography. Alpha Omegan 2010;103(2): 62-7.

4. World Medical Association. Declaration of Helsinki Ethical Principles for Medical Research involving human subjects; 2008.

5. White SC, Pharoah MJ. The evolution and application of dental maxillofacial imaging modalities. Dental Clinics of North America 2008;52(4):689-705.

6. The SEDENTEX CT project. Radiation Protection: Cone Beam CT for Dental and Maxillofacial Radiology. Provisional Guidelines, 2009.

7. Ludlow J B, Ivanovic M. Comparative dosimetry of dental CBCT devices and 64-slice CT for oral and maxillofacial radiology. Oral Surgery, Oral Medicine, Oral Pathology, Oral Radiology and Endodontology 2008;106(1):106-14.

8. Cotton TP, Geisler TM, Holden D T, Schwartz SA, Schindler WG. Endodontic applications of conebeam volumetric tomography. Journal of Endodontics 2007;33(9):1121-32.

9. De Paula-Silva FW, Wu MK, Leonardo M R, da Silva L A, Wesselink PR. Accuracy of periapical radiography and cone-beam computed tomography scans in diagnosing apical periodontitis using histopathological findings as a gold standard. Journal of Endodontics 2009;35(7): 1009-12.

10. Estrela C, Bueno MR, Leles CR, Azevedo B, Azevedo, JR. Accuracy of cone beam computed tomography and panoramic and periapical radiography for detection of apical periodontitis. Journal of Endodontics 2008;34(3):273-9.

11. Hassan B, Metska ME, Ozok AR, van der Stelt P, Wesselink PR. Detection of vertical root fractures in endodontically treated teeth by a cone beam computed tomography scan. Journal of Endodontics 2009;35(5):719-22.

12. Jafarzadeh H, Abbott PV. Dilaceration: review of an endodontic challenge. Journal of Endodontics 2007;33(9):1025-30.

13. Ansari I, Maria R. Managing curved canals. Contemporary Clinical Dentistry2012;3(2):23741.

14. Malcic A, Jukic S, Brzovic V, Miletic I, Pelivan I, Anic I. Prevalence of root dilaceration in adult dental patients in Croatia. Oral Surgery, Oral 
Medicine, Oral Patholology, Oral Radiology and Endodontology 2006;102(1):104-9.

15. Vertucci F J. Root canal anatomy of the human permanent teeth. Oral Surgery, Oral Medicine, Oral Pathology, Oral Radiology 1984;58(5):589-99.

16. Jafarzadeh H, Azarpazhooh A, Mayhall J T. Taurodontism: a review of the condition and endodontic treatment challenges. International Endodontic Journal 2008;41(5):375-88.

17. Jafarzadeh H, Wu YN. The C-shaped root canal configuration: a review. Journal of Endodontics 2007;33(5):517-23.

18. Friedlander LT, Cullinan M P, Love RM. Dental stem cells and their potential role in apexogenesis and apexification. International Endodontic Journal 2009;42(11):955-62.

19. Patel S, Dawood A, Wilson R, Horner K, Mannocci F. The detection and management of root resorption lesions using intraoral radiography and cone beam computed tomography an in vivo investigation. International Endodontic Journal 2009;42(9):831-8.

20. Patel S, Kanagasingam, S, Pitt Ford T. External cervical resorption: a review. Journal of Endodontics 2009;35(5):616-25.

21. Patel S, Ricucci D, Durak C, Tay F.Internal root resorption: a review. Journal of Endodontics 2010; 36(7):1107-21.

22. Friedman S, Abitbol S, Lawrence H P. Treatment outcome in endodontics: the Toronto Study. Phase 1: initial treatment. Journal of Endodontics 2003;29(12):787-93.

23. Orstavik D, Kerekes K, Eriksen HM. The periapical index: a scoring system for radiographic assessment of apical periodontitis. Endodontics E Dental Traumatology 1986;2(1):20-34.

24. Gröndahl HG, Huumonen S. Radiographic manifestations of periapical inflammatory lesions. Endodontic Topics 2004;8(1):55-67.

25. Rigolone M, Pasqualini D, Bianchi L, Berutti E, Bianchi S D. Vestibular surgical access to the palatine root of the superior first molar: "low-dose cone-beam" CT analysis of the pathway and its anatomic variations. Journal of Endodontics 2003; 29(11):773-5.

26. Molven O; Halse A; Fristad I. Long-term reliability and observer comparisons in the radiographic diagnosis of periapical disease. International Endodontic Journal 2002;35:142-7.

27. Estrela C, Bueno MR, Azevedo BC, Azevedo JR, Pecora JDA. new periapical index based on cone beam computed tomography. Journal of Endodontics 2008;34(11):1325-31.

28. Patel S, Wilson R, Dawood A, Mannocci F. The detection of periapical pathosis using periapical radiography and cone beam computed tomography, part 1: preoperative status. International Endodontic Journal 2012;45(8):702-10.

29. Kaya S, Yavuz I, Uysal I, Akkuş. Measuring bone density in healing periapical lesions by using cone beam computed tomography: a clinical investigation. Journal of Endodontics 2012;38(1):28-31.

\section{CORRESPONDENCIA}

Héctor Monardes Cortés

Cumming, 40

Santiago, Chile

Correo electrónico: hector.monardes@uss.cl 